\title{
Alpha-synucleinopathy and neuropsychological symptoms in a population-based cohort of the elderly
}

Julia Zaccai ${ }^{1}$, Carol Brayne ${ }^{1}$, Fiona E Matthews ${ }^{2}$, Paul G Ince ${ }^{3^{*}}$ and on behalf of the MRC Cognitive Function and Ageing Neuropathology Study

\begin{abstract}
Introduction: Studies with strong selection biases propose that alpha-synucleinopathy (AS) spreads upwards and downwards in the neuraxis from the medulla, that amygdala-dominant AS is strongly associated with Alzheimer's disease (AD), and that a more severe involvement of the cerebral cortex is correlated with increasing risk of dementia. This study examines the association of AS patterns and observed neuropsychological symptoms in brains of a population-representative donor cohort.

Methods: Brains donated in 2 out of 6 cognitive function and ageing study cohorts (Cambridgeshire and Nottingham) were examined. Over $80 \%$ were $>80$ years old at death. The respondents were evaluated prospectively in life for cognitive decline and dementia. Immunocytochemistry for tau and alpha-synuclein (using LB509 by Zymed Laboratories) was carried out in 208 brains to establish Braak stage and the pattern and severity of AS following the dementia with Lewy bodies (DLB) consensus recommendations. Dementia, specific neuropsychological measures as measured using the Cambridge cognitive examination, the presence of hallucinations and Parkinson's disease were investigated.
\end{abstract}

Results: Four patterns of AS were observed: no AS pathology ( $n=92)$, AS pathology following the DLB consensus guidelines ( $n=33$, of which five were 'neocortical'), amygdala-predominant AS $(n=18)$, and other AS patterns $(n=33)$. Each group was subdivided according to high/low neurofibrillary tangles (NFT) Braak stage. Results showed no association between dementia and these patterns of AS, adjusting for the presence of NFT or not. The risk of visual hallucinations shows a weak association with $A S$ in the substantia nigra (odds ratio $(O R)=3.2 ; 95 \%$ confidence interval (Cl) 0.5 to $15.5 ; P=0.09$ ) and amygdala $(\mathrm{OR}=3.0 ; 95 \% \mathrm{Cl} 0.7$ to $12.3 ; P=0.07)$. The analysis is similar for auditory hallucinations in subcortical regions.

Conclusions: Among the whole population of older people, AS does not increase the risks for dementia, irrespective of Braak stage of NFT pathology. There was no evidence that the pattern of AS pathology in cortical areas was relevant to the risk of hallucination. In general, the hypothesis that AS as measured using these methods per se is a key determinant of cognitive clinical phenotypes is not supported.

\footnotetext{
* Correspondence: p.g.ince@sheffield.ac.uk

${ }^{3}$ Sheffield Institute for Translational Neuroscience, 385A Glossop Road, Sheffield S10 2HQ, UK

Full list of author information is available at the end of the article
} 


\section{Introduction}

Diagnostic protocols for the neuropathological assessment of the ageing brain, for use in both research and clinical diagnosis, are based on the severity and anatomical distribution of lesions [1-3]. There is an expectation that 'cases' will be pathologically distinguishable from unimpaired individuals although the pathologies of Alzheimer's disease (AD), Parkinson's disease (PD) and Dementia with Lewy bodies (DLB) are routinely present in normal elderly brain donors [4-8]. Comparison of brain pathology in AD cases with DLB and PD cases suggests that Lewy body (LB) pathology contributes significantly to dementia [9-13]. However, there is no evidence-based consensus for a threshold of AS that will distinguish demented from non-demented people $[5,14,15]$. The prevalence of $\alpha$-synucleinopathy (AS), the substrate of LB pathology, is reported to vary between $2 \%$ to $12 \%$ in people without dementia at death and from $12 \%$ to $61 \%$ in people with dementia $[5,14,16-18]$. Limited AS in cortical and subcortical areas is variably interpreted as presymptomatic disease $[7,19]$ or non-specific age-related degeneration.

This previous work on the clinicopathological association of alpha-synucleinopathy is based on cohorts derived from secondary referral, memory clinics or community volunteers. These methods of selection introduce underestimated biases that prevent extrapolation of the conclusions to the whole population of elderly people. In general the impact of pathology is exaggerated in such studies [20]. In this study the association between AS, AD pathology, dementia, clinical PD and neuropsychological symptoms was assessed in a truly population-representative sample free of selection biases associated with clinical diagnosis, symptoms or access to health and social care facilities.

\section{Methods \\ Medical Research Council Cognitive Function and Ageing Study}

The Medical Research Council Cognitive Function and Ageing Study (MRC CFAS) has been fully described elsewhere [21]. It is a longitudinal, prospective, populationbased cohort study initiated in 1989. At each of five centres in England and Wales random samples of about 2,500 people, 64-years old and older (with an 82\% response rate), agreed to a structured initial interview. Structured interviews collected details of sociodemographic status, general health, Mini Mental State Examination (MMSE) [22] and functional ability. Approximately $20 \%$ of the sample completed a more detailed diagnostic assessment, including full mood and organicity sections of the Geriatric Mental State (GMS) Automated Geriatric Examination for Computer Assisted Taxonomy (AGECAT) [23] and the Cambridge Cognitive Examination (CAMCOG) [24].
Further waves of interviewing (both screening and assessment) were conducted at two, five and nine years after baseline on the survivors. In a sixth centre 5,200 people, 65 -years old and older, were interviewed using the same assessment interviews with follow-up at one to two, three to four, five to six, and nine years later. The CFAS Neuropathology Study is based on a brain donor programme established in 1992. Individuals selected for the assessment interviews were approached by a trained liaison officer for brain donation. The donation programme was discussed with the respondent and their family or carer as appropriate. All participants in the study gave their informed consent. This analysis is based on the burden and anatomical distribution of AS in all donations from two MRC CFAS centres before July 2003 (Nottingham and Cambridgeshire, $\mathrm{n}=208$ ). Research was carried out after approval from the Eastern Multi Centre Research Ethics Committee based at Papworth Hospitals NHS Trust (reference 03/5/017).

\section{Postmortem procedure and anatomical sampling}

Pathological evaluation of the Neuropathology Study cohort had previously been carried out using the Consortium to Establish a Registry for Alzheimer's Disease (CERAD) protocol [1] and each brain was assigned a Braak score for neurofibrillary tangles [2]. At autopsy samples of brain tissue were removed for frozen storage. The remainder of the brain was fixed for standardised assessment using paraffin-embedded tissues. For the evaluation of synucleinopathy a hierarchical sampling strategy was adopted based on evaluation of the midbrain (substantia nigra), medulla (dorsal efferent nucleus of the vagus nerve, medullary reticular formation) and amygdala to detect AS pathology. This method of screening was selected because literature at the time of the study indicated that no cases had been reported in which AS was present in any brain region but absent from these three screening areas [3,25-29]. If at least one positive $\alpha$-synuclein immunoreactive profile was found in a screening area five additional areas were investigated (cingulate gyrus, entorhinal cortex and hippocampus, frontal cortex, parietal cortex and temporal cortex), comprising the DLB Consensus recommendations for the evaluation of AS [30].

\section{Immunocytochemistry for alpha-synuclein, quantification}

Microwave antigen retrieval (citric acid $10 \mathrm{mM}$ pH6) of $6 \mu \mathrm{m}$ brain sections was followed by immunocytochemistry using a monoclonal antibody to mouse alphasynuclein (LB509, Zymed Laboratories Inc; San Francisco, CA USA; dilution $1 / 200$; incubated at $4^{\circ} \mathrm{C}$ overnight). Incubation with biotinylated secondary antibody $(1 / 250)$ for 1.5 hours at room temperature was followed by ABC Elite Mouse IgG reagents (Vector Laboratories, Burlingame, CA, USA) for one hour. Reaction product was visualised 
using Vector Nova Red (Vector Laboratories) according to the manufacturer's protocol. All sections were counterstained with haematoxylin and mounted in DPX. Interpretation of the appearances was jointly established with propidium iodide (PI). Acceptable inter- and intra-rater reliability was previously reported in this study [31]. AS pathology was then rated by a single investigator (JZ). The microscopic field with the highest density of AS was identified and the number of AS profiles was counted at x200 magnification. Spherical and densely stained structures were considered to be LB irrespective of intra- or extracellular localisation. Elongated AS-positive profiles were considered to be Lewy neurites (LN). Two consecutive sections from each block were assessed and the highest density score retained for the statistical analysis. In the final analysis, results were dichotomised to AS present versus absent.

Each brain was allocated to a pathological category as shown in Table 1. These categories were based on the presence or absence of neurofibrillary tangles (NFT) and AS. NFT pathology was dichotomised on the basis of Braak stage (Low - stages I-III versus High - stages IV-VI). AS was divided into three categories based on previous work in this cohort:

1. Cases that conform to the hierarchy of anatomical spread of AS implicit in the DLB Consensus protocol for the evaluation of AS [30], which also implies that these cases conform to the Braak staging hypothesis proposed for AS [3]. Some analyses are based on the stratification of this group into those with DLB 'limbic' and 'neocortical' disease and those with AS confined to midbrain/brainstem structures;
2. Cases that conform to 'amygdala-predominant' AS implying that amygdala AS is prominent and that involvement of neocortical, brainstem and midbrain structures is limited and patchy [31];

3. Cases with an atypical AS distribution, predominantly those with mild to severe cortical involvement in the absence of AS in one of the lower hierarchic anatomical sites (for example, substantia nigra, locus ceruleus, dorsal vagus nucleus). For example, this category includes cases with prominent AS involvement observed in the substantia nigra and frontal cortex but not in other investigated areas, or cases with prominent AS involvement observed in the vagus nucleus and parietal cortex only. These also include six cases corresponding to the 'cerebral-form of DLB' [32] where AS was observed in the neocortex predominantly.

Previous findings in this cohort showed that there was no association between these different AS staining patterns and Braak stage for NFT [31]. Thirty-two brains were excluded from the final analysis due to missing pathological or clinical data.

\section{Dementia and other outcomes}

Dementia was defined using a combination of the AGECAT algorithm, death certificates and informant interviews, including retrospective informant interviews after death (RINI). Ten percent of participants were classified as having unknown dementia status on the basis that both the time between the last interview and death was too long to exclude incident dementia (over six months) and that data collected during informant interviews were inconclusive. The presence or absence of hallucinations

Table 1 Pathology subgroups based on a-synucleinopathy (AS) and severity of neurofibrillary tangle (NFT) pathology

\begin{tabular}{llll}
\hline Neuropathology group & & Criteria & Number \\
\hline No pathology & & No AS; Braak stages I-III NFT & 49 \\
NFT only & No AS; Braak stages IV-VI NFT & 43 \\
AS & At least 1 LB or LN in any region & 84 \\
AS & + high NFT & AS + Braak stages IV-VI NFT & 32 \\
AS & + low NFT & AS + Braak stages I-III NFT & 52 \\
AS DLB & + high NFT & AS pattern consistent with DLB Consensus; Braak stages IV-VI NFT & 13 \\
AS DLB & + low NFT & AS pattern consistent with DLB Consensus; Braak stages I-III NFT & 20 \\
AS Amygdala-predominant & + high NFT & amygdala-predominant AS pattern: Braak stages IV-VI NFT & 9 \\
AS Amygdala-predominant & + low NFT & amygdala-predominant AS pattern: Braak stages I-III NFT & 9 \\
AS Other pattern & + high NFT & AS pattern not consistent with DLB consensus or amygdala-predominant AS; & 10 \\
AS Other pattern & & Braak stages IV-VI NFT & 23 \\
& + low NFT & AS pattern not consistent with DLB consensus or amygdala-predominant AS: & 2
\end{tabular}

DLB, dementia with Lewy bodies. 
(visual and auditory) or PD was based on self-reported symptoms and informant interview data.

\section{Specific neuropsychological measures}

General cognitive function was assessed using the MMSE. A score of 21 or less was used to indicate the likely presence of dementia. The CAMCOG neuropsychological battery includes seven areas of cognition: orientation, language (comprehension and expression), memory (learning, recent and remote), attention/calculation, praxis, abstract thinking and perception. Cut-off scores for each domain subscales were taken as values falling below the 25th percentile cut-point in the whole of the nondemented MRC CFAS population. Results are presented for a total CAMCOG score (all seven areas) and for CAMCOG areas of attention/calculation, language (comprehension and expression), abstract thinking and perception. CAMCOG cut-off points were 83 out of a maximum score of 103, 5 out of 8 (attention/calculation), 25 out of 30 (language), 5 out of 8 (abstract thinking) and 6 out of 8 (perception). Informant and interview data were analysed for the presence or absence of visual hallucination, auditory hallucination and a self-reported diagnosis of Parkinson's disease.

\section{Statistical analysis}

MRC CFAS has released data at defined points. For this study, version 8.2 of the core MRC CFAS dataset and version 3.3 of the neuropathology dataset were used. All statistical work was conducted in Stata Software version 9.0 (Stata Corporation, College Station, TX, USA). Odds ratios and Fischer's exact (chi squared) tests were applied to estimate the strength of any association between two observations. High $P$-values are associated with a higher likelihood that there is no significant difference between compared groups. All $P$-values are two sided.

\section{Results}

\section{Demographics and AS staging patterns of the study} cohort

The sample used in this analysis is representative of all MRC CFAS centres (Table 2). It includes a wide range of age-related neuropathology and a spectrum of clinical states including dementia and normal cognition. The sample contains a high proportion of the 'oldest-old' in whom the burden of dementia and neurodegeneration is highest.

Among the 176 brain donors included in this analysis the four patterns of AS were distributed as follows: (1) no AS pathology ( $\mathrm{n}=92)$; (2) AS pathology following the

Table 2 Demographics of the respondents

\begin{tabular}{|c|c|c|c|c|c|c|c|c|c|c|}
\hline \multirow{2}{*}{$\begin{array}{l}\text { Demographic characteristic } \\
\text { Sex }\end{array}$} & \multicolumn{2}{|c|}{$\begin{array}{l}\text { All MRC CFAS } \\
\text { (Number }=451 \text { ) }\end{array}$} & \multicolumn{2}{|c|}{$\begin{array}{l}\text { Cambridgeshire and } \\
\text { Nottingham cohorts } \\
\text { (Number }=208 \text { ) }\end{array}$} & \multicolumn{2}{|c|}{$\begin{array}{c}\text { NFT only } \\
\text { (Number }=43 \text { ) }\end{array}$} & \multicolumn{2}{|c|}{$\begin{array}{c}\text { AS + NFT } \\
(\text { Number }=32)\end{array}$} & \multicolumn{2}{|c|}{$\begin{array}{c}\text { AS only } \\
\text { (Number= 52) }\end{array}$} \\
\hline & & & & & & & & & & \\
\hline Men & 188 & $(41)$ & 123 & (59) & 10 & (23) & 16 & $(50)$ & 21 & (40) \\
\hline Women & 268 & (59) & 85 & $(41)$ & 33 & (74) & 16 & $(50)$ & 31 & (60) \\
\hline \multicolumn{11}{|l|}{ Age at death } \\
\hline 70 to 79 & 89 & (19) & 40 & (19) & 5 & $(12)$ & 4 & $(12)$ & 15 & (29) \\
\hline 80 to 89 & 204 & (49) & 102 & $(49)$ & 19 & (44) & 16 & $(50)$ & 28 & (54) \\
\hline 90 to 105 & 163 & $(32)$ & 66 & $(32)$ & 19 & (44) & 12 & $(48)$ & 9 & (17) \\
\hline \multicolumn{11}{|l|}{ Education } \\
\hline$<10$ years & 318 & (69) & 159 & $(76)$ & 33 & (77) & 26 & $(81)$ & 35 & (67) \\
\hline $10+$ years & 138 & (31) & 49 & $(24)$ & 10 & (23) & 6 & (19) & 17 & (33) \\
\hline \multicolumn{11}{|l|}{ Social class } \\
\hline Non manual & 165 & $(36)$ & 79 & (38) & 17 & (39) & 12 & $(37)$ & 17 & (33) \\
\hline Manual & 234 & $(51)$ & 110 & (33) & 21 & (49) & 16 & $(50)$ & 30 & (58) \\
\hline Unclassified/Army & 57 & (13) & 19 & (9) & 5 & $(12)$ & 4 & (13) & 5 & (9) \\
\hline \multicolumn{11}{|l|}{ Dementia status $^{a}$} \\
\hline Dementia & 242 & (53) & 105 & $(50)$ & 30 & (70) & 24 & $(75)$ & 18 & (34) \\
\hline No dementia & 184 & $(40)$ & 83 & $(40)$ & 12 & (28) & 6 & (19) & 27 & (53) \\
\hline Unknown ${ }^{b}$ & 30 & (7) & 20 & $(10)$ & 1 & $(2)$ & 2 & (6) & 7 & (13) \\
\hline
\end{tabular}

${ }^{a}$ Determined using AGECAT organicity scores, informants interview items and death certificates; ${ }^{b}$ unknown dementia status was assigned on the basis that both the time between the last interview and death was too long to exclude incident dementia (over six months) and the data collected from interviews were inconclusive. AS, alpha-synucleinopathy; MRC CFAS, Medical Research Council Cognitive Function and Ageing Study; NFT, neurofibrillary tangles. 
DLB Consensus guidelines ( $\mathrm{n}=33$; of which 5 were 'neocortical'); (3) amygdala-predominant AS ( $\mathrm{n}=18)$ [28]; (4) other AS patterns $(n=33)$. Each group was subdivided according to the presence of NFT as defined by NFT Braak stage as dichotomised to low or high score (Table 1).

\section{AS and dementia or features of DLB}

LB pathology was found in 34\% of those without dementia during life and in $45 \%$ of those with dementia. The odds ratio for risk of dementia in association with LB is 1.6 ( $95 \%$ confidence interval 0.8 to $3.0, P=0.1$ ). There is a significant association between self-reported Parkinson's disease and the presence of AS in substantia nigra, amygdala and the parietal neocortex (Table 3). There is also weak evidence of involvement of the cingulate cortex. These findings are consistent with the expected associations from the literature. The risk of visual hallucination in this population cohort shows weak evidence of an association with AS in substantia nigra $(\mathrm{OR}=3.2$; $95 \%$ CI 0.5 to 15.5 ; $P=0.09)$ and amygdala $(\mathrm{OR}=3.0 ; 95 \% \mathrm{CI} 0.7$ to 12.3 ; $P=0.07$ ). The analysis is similar for auditory hallucinations in subcortical regions. The odds ratios for visual hallucinations are higher in cortical regions compared to those for auditory hallucination but the small sample size restricts the statistical power of this analysis.

\section{Association between AS staging pattern and neuropsychological symptoms}

An initial analysis (not shown) compared the association of cognitive outcome variables between cases with neocortical and limbic patterns of AS according to the DLB Consensus guidelines and those with only brainstem involvement. In this population-based sample, none of the outcomes were significantly different for these two groups and they were combined in the analysis as the 'DLB Consensus pattern' group. Odds ratios for the association between neuropsychological symptoms and the different pathology groups are presented in Table 4. Adjusting for age, education, the presence of any vascular pathology, or a CAMCOG assessment performed more than two years before death, did not change the results. There was no association between AS and dementia $(\mathrm{OR}=0.88$; $95 \%$ CI 0.35 to 2.22 ) or between AS and low MMSE in cases with low NFT Braak stage $(\mathrm{OR}=0.96$; $95 \%$ CI 0.41 to 2.26 ). There was no additional risk of cognitive impairment when comparing brains with combined AS and NFT (OR for dementia $=4.63 ; 95 \%$ CI 1.52 to 15.1 ) to brains with NFT only (OR $=3.68$; $95 \%$ CI 1.37 to 10.08). Among the three AS pathology groups with low NFT Braak stage, the group conforming to DLB Consensus guidelines was more likely to be demented $(\mathrm{OR}=3.24$; 95\% CI 0.87 to 13.47). Brains classified as 'neocortical' DLB by the DLB Consensus guidelines, irrespective of NFT $(n=5)$, included only two with dementia. None of the nine brains with amygdala-predominant AS in the absence of NFT had dementia. These sub-groups are too small for statistical analysis. The cases with AS only in neocortical areas ('cerebral form of DLB'; $n=6$ ) [32], irrespective of NFT Braak stage, had a high likelihood of being demented (five of six), and all had low MMSE scores. However, there was no evidence of an association between the presence of this 'cerebral-form of DLB' and individual domains of CAMCOG scores. Braak stage in the five demented cases was: 0 (one case); 3 (one case); 5 (two cases); 6 (one case).

Analysis of the risk associated with sub-threshold performance in individual domains of CAMCOG were not shown to be elevated above the control 'no pathology' group even in cases with high NFT Braak stage or combined AS and high NFT Braak stage. This reflects the sample sizes and the likely lack of power to detect an effect. It is interesting that in the AS cases with low NFT Braak stage the risk of subthreshold performance in the perceptual domain was increased for those with amygdala predominant disease $(\mathrm{OR}=3.11 ; 95 \% \mathrm{CI} 0.32$ to 153.1) and the atypical AS group $(\mathrm{OR}=4.15 ; 95 \% \mathrm{CI}$ 0.77 to 41.44 ) but the robustness of this finding needs to be confirmed in a larger study.

\section{Discussion}

We report the clinicopathological correlation of AS in a cohort of brains from older people donated from a population-based study. In this cohort of CFAS respondents, a reliable diagnosis of cognitive state at death was possible in 90\%: 40\% were non-demented at death and $50 \%$ demented. AS was present in $39 \%$ of the 208 brains examined. We have previously shown that AS in the population does not always conform to the DLB Consensus model of hierarchic spread, or the similar model implicit in proposed Braak staging of AS [3,30,31]. Only half of the brains with AS in this sample conform to DLB Consensus or Braak staging. A further 29\% conform to the more recent concept of amygdala-predominant AS $[33,34]$. However, one in five old people with AS show a pattern that is not typical of either of these models, including approximately 3\% who have AS largely restricted to the neocortex.

Previous studies reporting on a relationship between AS and neurologic and neuropsychological outcomes generally make a number of assumptions. These include a systematic hierarchical spread of disease upwards and downwards in the neuraxis from the medulla, a strong association between amygdala-predominant AS and Alzheimer's disease, and the expectation that more severe involvement of the cerebral cortex is correlated with increasing risk of dementia [2,25-29,31,33-37]. There is also emerging evidence that $\mathrm{AS}$ is present in aged brains without neuro-psychiatric disorders or extra-pyramidal 
Table 3 Relationship between a-synucleinopathy (AS) and a diagnosis of Parkinson's disease (PD), visual hallucinations (VH) and auditory hallucinations (AH) Brain area $\%$ (brains showing AS)

\begin{tabular}{|c|c|c|c|c|c|c|c|c|c|c|c|c|}
\hline & \\
\hline & No PD $(n=201)$ & $\mathrm{PD}(\mathrm{n}=7)$ & OR $(95 \% \mathrm{Cl})$ & $P$ & No VH $(n=196)$ & $\mathrm{VH}(\mathrm{n}=12)$ & OR $(95 \% \mathrm{Cl})$ & $P$ & No $A H(n=194)$ & $\mathrm{AH}(\mathrm{n}=14)$ & OR $(95 \% \mathrm{Cl})$ & $P$ \\
\hline Substantia nigra & 11 & 50 & 7.8 (1.0 to 60.6$)$ & $<0.01$ & 12 & 30 & $3.2(0.5$ to 15.5$)$ & 0.09 & 11 & 31 & 3.5 (0.7 to 13.9$)$ & 0.04 \\
\hline Vagus nucleus & 21 & 43 & 2.9 (0.4 to 17.8$)$ & $>0.2$ & 21 & 25 & 1.2 (0.2 to 5.3$)$ & $>0.2$ & 22 & 36 & 2.2 (0.5 to 7.8$)$ & $>0.2$ \\
\hline Amygdala & 22 & 57 & 4.7 (0.7 to 33.4$)$ & 0.03 & 22 & 45 & 3.0 (0.7 to 12.3 ) & 0.07 & 22 & 36 & $1.9(0.5$ to 6.9$)$ & $>0.2$ \\
\hline Frontal cortex & 2 & 0 & & & 2 & 0 & & & 2 & 0 & & \\
\hline Temporal cortex & 2 & 0 & & & 2 & 8 & 4.0 (0.1 to 44.2$)$ & $>0.2$ & 2 & 0 & & \\
\hline Parietal cortex & 3 & 29 & 11.1 (0.9 to 82.3) & $<0.01$ & 4 & 8 & 2.1 (0.04 to 18.5$)$ & $>0.2$ & 4 & 7 & $1.8(0.04$ to 15.2$)$ & $>0.2$ \\
\hline Hippocampus & 2 & 0 & & & 2 & 0 & & & 2 & 0 & & \\
\hline Entorhinal cortex & 6 & 0 & 2.1 (0.04 to 18.2$)$ & $>0.2$ & 7 & 0 & & & 6 & 7 & 1.2 (0.02 to 9.1$)$ & $>0.2$ \\
\hline Cingulate cortex & 3 & 14 & $5.4(0.1$ to 57.2$)$ & $>0.2$ & 3 & 8 & $2.9(0.05$ to 27.1$)$ & $>0.2$ & 3 & 7 & 2.4 (0.05 to 22.2 ) & $>0.2$ \\
\hline
\end{tabular}

$\mathrm{Cl}$, confidence interval; OR, odds ratio. 
Table 4 Association between neuropsychological symptoms and pathology groups (when compared to associations with a no pathology group, unadjusted)

\begin{tabular}{|c|c|c|c|c|c|c|c|c|c|c|c|}
\hline \multirow[t]{2}{*}{ Neuropsychological measure } & \multicolumn{4}{|c|}{ High NFT only } & \multicolumn{4}{|c|}{ AS + high NFT } & \multicolumn{3}{|c|}{ AS + low NFT } \\
\hline & $\bar{n}$ & $\mathrm{~N}$ & OR $95 \% \mathrm{Cl}$ & $P$ & $\bar{n}$ & $\mathbf{N}$ & OR $95 \% \mathrm{Cl}$ & $P$ & $n$ & $\mathbf{N}$ & OR $95 \% \mathrm{Cl}$ \\
\hline MMSE score $<21$ & 32 & 43 & $3.53(1.30-10.01)$ & $<0.01$ & 23 & 32 & $2.21(0.81-6.27)$ & $>0.2$ & 25 & 52 & $0.96(0.41-2.26)$ \\
\hline Dementia & 30 & 43 & $3.68(1.37-10.08)$ & $<0.01$ & 24 & 32 & $4.63(1.52-15.07)$ & $<0.01$ & 17 & 52 & $0.88(0.35-2.22)$ \\
\hline Low CAMCOG Attention & 25 & 43 & $1.29(0.41-4.27)$ & $>0.2$ & 15 & 32 & $0.69(0.21-2.32)$ & $>0.2$ & 23 & 52 & $0.56(0.20-1.54)$ \\
\hline Low CAMCOG Language ${ }^{a}$ & 27 & 43 & $2.05(0.56-8.46)$ & $>0.2$ & 21 & 32 & $1.99(0.49-9.68)$ & $>0.2$ & 26 & 52 & $0.7(0.24-2.02)$ \\
\hline Low CAMCOG Abstract thinking & 18 & 43 & $1.93(0.68-5.51)$ & $>0.2$ & 13 & 32 & $1.62(0.53-5.02)$ & $>0.2$ & 20 & 52 & $1.5(0.57-4.00)$ \\
\hline Low CAMCOG Perception & 27 & 43 & $2.33(0.70-8.47)$ & $>0.2$ & 16 & 32 & $0.83(0.27-2.63)$ & $>0.2$ & 31 & 52 & $2.01(0.66-6.39)$ \\
\hline \multirow[t]{2}{*}{ Low CAMCOG Total ${ }^{\text {b }}$} & 29 & 43 & $3.67(0.83-22.20)$ & $>0.2$ & 19 & 32 & $1.44(0.38-6.13)$ & $>0.2$ & 26 & 52 & $0.82(0.28-2.43)$ \\
\hline & \multicolumn{4}{|c|}{ DLB Consensus + high NFT } & \multicolumn{4}{|c|}{ Amygdala-predominant + high NFT } & \multicolumn{3}{|c|}{ other AS pattern + high NFT } \\
\hline MMSE score $<21$ & 10 & 13 & $3.21(0.70-19.89)$ & $>0.2$ & 7 & 9 & $2.24(0.44-14.76)$ & $>0.2$ & 6 & 10 & $1.44(0.30-7.77)$ \\
\hline Dementia & 10 & 13 & $4.5(0.96-28.04)$ & 0.06 & 7 & 9 & $4.72(0.77-49.99)$ & 0.07 & 7 & 10 & $4.72(0.77-49.99)$ \\
\hline Low CAMCOG Attention & 7 & 13 & $1.45(0.23-16.15)$ & $>0.2$ & 4 & 9 & $0.41(0.07-2.66)$ & $>0.2$ & 4 & 10 & $0.55(0.08-4.40)$ \\
\hline Low CAMCOG Language ${ }^{a}$ & 9 & 13 & $3.41(0.38-163.00)$ & $>0.2$ & 7 & 9 & $2.66(0.28-130.36)$ & $>0.2$ & 5 & 10 & $0.95(0.13-11.36)$ \\
\hline Low CAMCOG Abstract thinking & 4 & 13 & $1.2(0.20-6.54)$ & $>0.2$ & 5 & 9 & $1.88(0.34-10.87)$ & $>0.2$ & 4 & 10 & $2(0.29-15.30)$ \\
\hline Low CAMCOG Perception & 8 & 13 & $2.07(0.34-22.35)$ & $>0.2$ & 5 & 9 & $0.65(0.12-3.85)$ & $>0.2$ & 3 & 10 & $0.39(0.05-2.71)$ \\
\hline \multirow[t]{2}{*}{ Low CAMCOG Total ${ }^{b}$} & 8 & 13 & $3.03(0.33-146.68)$ & $>0.2$ & 5 & 9 & $0.63(0.10-4.81)$ & $>0.2$ & 6 & 10 & $2.28(0.23-114.05)$ \\
\hline & \multicolumn{4}{|c|}{ DLB Consensus + low NFT } & \multicolumn{4}{|c|}{ Amygdala-predominant + low NFT } & \multicolumn{3}{|c|}{ other AS pattern + low NFT } \\
\hline MMSE score <21 & 13 & 20 & $1.39(0.45-4.38)$ & $>0.2$ & 1 & 9 & $0.12(0.00-1.04)$ & $>0.2$ & 11 & 23 & $1.32(0.40-4.46)$ \\
\hline Dementia & 12 & 20 & $3.24(0.87-13.47)$ & 0.09 & 0 & 9 & $0(0.00-0.62)$ & $>0.2$ & 5 & 23 & $0.56(0.13-2.09)$ \\
\hline Low CAMCOG Attention & 10 & 20 & $0.83(0.20-3.78)$ & $>0.2$ & 1 & 9 & $0.07(0.00-0.70)$ & $>0.2$ & 12 & 23 & $0.83(0.22-3.35)$ \\
\hline Low CAMCOG Language ${ }^{a}$ & 9 & 20 & $0.57(0.14-2.45)$ & $>0.2$ & 5 & 9 & $0.95(0.13-11.36)$ & $>0.2$ & 12 & 23 & $0.76(0.20-3.11)$ \\
\hline Low CAMCOG Abstract thinking & 6 & 20 & $1(0.24-3.91)$ & $>0.2$ & 2 & 9 & $0.6(0.05-4.29)$ & $>0.2$ & 12 & 23 & $3(0.82-11.68)$ \\
\hline Low CAMCOG Perception & 9 & 20 & $0.93(0.23-4.26)$ & $>0.2$ & 6 & 9 & $3.11(0.32-153.14)$ & $>0.2$ & 16 & 23 & $4.15(0.77-41.44)$ \\
\hline Low CAMCOG Total ${ }^{b}$ & 7 & 20 & $0.44(0.10-2.01)$ & $>0.2$ & 6 & 9 & $2.28(0.23-114.05)$ & $>0.2$ & 13 & 23 & $0.99(0.25-4.38)$ \\
\hline
\end{tabular}

${ }^{a}$ Comprehension and Expression; ${ }^{b}$ over seven CAMCOG domains. AS, alpha-synucleinopathy; CAMCOG, Cambridge Cognitive Examination; Cl, confidence interval; DLB, dementia with Lewy bodies; MMSE, Mini Mental State Examination; NFT, neurofibrillary tangles; OR, odds ratio.

symptoms $[6,7,38]$. These variations can be explained by the fact that the research studies that underpin these assumptions did not involve the assessment of true population-based cohorts so that they emerged from, and have been tested in, cohorts influenced by selection biases associated with clinical referral, clinical case selection, hospitalisation or volunteer recruitment strategies, and in addition, to selection related to the pathological findings. The possibility therefore arises that inter-individual variation in pathophysiological responses might account for the differences seen between disease-based and population-based observations. It is thus possible that individuals who develop the clinicopathological features of a Lewy Body disorder (for example, PD and DLB) are predisposed to enable hierarchic spread of synucleinopathy in the brain whereas those chiefly encountered in population-based samples, and large unselected autopsy series, with atypical distribution of pathology and absent clinical features somehow resist such propagation.
In the present analysis we show that, among the whole population of elderly people, AS does not increase the overall risk for dementia, nor low MMSE or low CAMCOG scores, irrespective of whether it is associated with high or low Braak stage of NFT pathology. In AS cases with a low NFT Braak stage, the group showing pathological spread of AS consistent with the anatomical hierarchy implicit in the DLB Consensus and Braak staging, had an increased risk of dementia $(\mathrm{OR}=3.24 ; 95 \% \mathrm{CI}$ 0.87 to 13.47$)$ compared to the amygdala-predominant $(\mathrm{OR}=0 ; 95 \% \mathrm{CI} 0.0$ to 0.62$)$ and atypical AS groups $(\mathrm{OR}=0.56 ; 95 \% \mathrm{CI} 0.13$ to 2.09$)$. This observation may in part explain the much more robust correlations with cognitive status reported in many cohort studies that largely concentrate on this subgroup of synucleinopathy. In addition, the cases with the so-called 'neocortical-form of DLB' were very likely to be demented (five of six), although three of these five cases had a high NFT Braak stage. Nonetheless, the overall risk of dementia in AS was not significantly associated with cortical compared to 
subcortical pathology. There was no relationship between any of the domains of cognition tested within the CAMCOG battery and AS in specific brain regions either with high or low NFT Braak stages. There was an expected relationship between self-reported PD and AS in the substantia nigra although self-reporting of PD in MRC CFAS, albeit suitable as a screening tool, was also found to identify a heterogeneous group of movement disorder patients [39]. This relationship also extended to the amygdala and parietal cortex with some evidence in the cingulate cortex. This latter association needs to be tested again with greater power or by using a different approach. These data reflect the probable greater concordance of the Braak staging model in the group with a clinicopathological diagnosis of Lewy body PD since the hypothesis was developed through the study of that disease together with two cohorts derived from secondary referrals selected on the basis of either AS pathology in the absence of known clinical disorder or absence of AS pathology in the brainstem with no clinical record of neurological disease [3]. Finally, there was evidence for an excess of visual hallucination in association with AS compared to auditory hallucination but both were associated with substantia nigra involvement. There was no evidence that the pattern of AS pathology was relevant to the risk of hallucination and no relationship with cortical pathology.

Although this is a relatively large cohort, the population sampling frame means that the power of the study is more limited in assessing the relationship between AS and specific neuropsychological features because the sample is not enriched either for cases with more extensive AS involving cortical regions or for specific clinical features such as hallucination. Another point that limits the conclusions of this study is that AS may have been underestimated in this group as its pathology in the olfactory bulb was not included in the initial screening process [40]. There is also an inherent underestimation of AS as a result of the study design in that solely two consecutive sections from each area were included in the assessment process. However, cortical pathology is present, and for most clinical correlates equally prevalent, in cases with and without these specific features.

\section{Conclusions}

In general the hypothesis that AS per se is a key determinant of cognitive clinical phenotypes is not supported in this rare study of clinicopathological correlations of AS in a cohort of brains from older people donated from a population-based study.

\footnotetext{
Abbreviations

AD: Alzheimer's disease; AGECAT: automated examination for computer assisted taxonomy; AS: alpha-synucleinopathy; CAMCOG: Cambridge cognitive examination; CERAD: Consortium to Establish a Registry for Alzheimer's Disease; Cl: confidence interval; DLB: Dementia with Lewy
}

bodies; GMS: Geriatric Mental State; LB: Lewy bodies; MMSE: Mini Mental State Examination; MRC CFAS: Medical Research Council Cognitive Function and Ageing Study; NFT: neurofibrillary tangles; OR: odds ratio; PD: Parkinson's disease; RINI: Retrospective informant interviews after death.

\section{Competing interests}

The authors declare that they have no competing interests.

\section{Authors' contributions}

JZ carried out the immunocytochemistry, rated the alpha-synuclein pathogy, performed the statistical analysis and drafted the manuscript. FM participated in the design of the study and performed the statistical analysis. CB and PI conceived the study, participated in its design and coordination and helped draft the manuscript. All authors read and approved the final manuscript.

\section{Acknowledgments}

The authors thank Gill Forster, Catherine Gelsthorpe and Lynne Baxter for their assistance with the immunohistochemistry, and Sally Hunt for helping with the inter-rater validity testing. JZ received Medical Research Council PhD and Newton European Research Studentships and a Wingate Foundation Scholarship. Further information can be found on www.cfas.ac.uk.

\section{Author details}

${ }^{1}$ Institute of Public Health, University of Cambridge, Cambridge, UK. ${ }^{2}$ MRC Biostatistics Unit, Institute of Public Health, Cambridge, UK. ${ }^{3}$ Sheffield Institute for Translational Neuroscience, 385A Glossop Road, Sheffield S10 2HQ, UK.

Received: 30 May 2014 Accepted: 27 January 2015

Published online: 13 April 2015

\section{References}

1. Mirra SS, Heyman A, McKeel D, Sumi SM, Crain BJ, Brownlee LM, et al. The Consortium to Establish a Registry for Alzheimer's Disease (CERAD). Part II. Standardization of the neuropathologic assessment of Alzheimer's disease. Neurology. 1991;41:479-86.

2. Braak H, Braak E. Staging of Alzheimer's disease-related neurofibrillary changes. Neurobiol Aging. 1995;16:271-8.

3. Braak H, Del Tredici K, Rub U, de Vos RAl, Jansen Steur ENH, Braak E. Staging of brain pathology related to sporadic Parkinson's disease. Neurobiol Aging. 2003;24:197-211.

4. Neuropathology Group of the Medical Research Council Cognitive Function and Ageing Study. Pathological correlates of late-onset dementia in a multicentre, community-based population in England and Wales. Lancet. 2001;357:169-75.

5. Wakisaka Y, Furuta A, Tanizaki Y, Kiyohara Y, lida M, Iwaki T. Age-associated prevalence and risk factors of Lewy body pathology in a general population: the Hisayama study. Acta Neuropathol. 2003;106:374-82.

6. Parkinen L, Pirttilä T, Alafuzoff I. Applicability of current staging/ categorization of alpha-synuclein pathology and their clinical relevance. Acta Neuropathol. 2008;115:399-407.

7. Jellinger KA. Lewy body-related alpha-synucleinopathy in the aged human brain. J Neural Transm. 2004;111:1219-35.

8. Horvath J, Herrmann FR, Burkhard PR, Bouras C, Kövari E. Neuropathology of dementia in a large cohort of patients with Parkinson's disease. Parkinsonism Relat Disord. 2013;19:864-8.

9. Haroutunian V, Serby M, Purohit DP, Perl DP, Marin D, Lantz M, et al. Contribution of Lewy body inclusions to dementia in patients with and without Alzheimer disease neuropathological conditions. Arch Neurol. 2000:57:1145-50.

10. Hurtig HI, Trojanowski JQ, Galvin J, Ewbank D, Schmidt ML, Lee VM, et al. Alpha-synuclein cortical Lewy bodies correlate with dementia in Parkinson's disease. Neurology. 2000;54:1916-21.

11. Mattila PM, Rinne JO, Helenius H, Dickson DW, Roytta M. Alpha-synucleinimmunoreactive cortical Lewy bodies are associated with cognitive impairment in Parkinson's disease. Acta Neuropathol. 2000;100:285-90.

12. Olichney JM, Galasko D, Salmon DP, Hofstetter CR, Hansen LA, Katzman R, et al. Cognitive decline is faster in Lewy body variant than in Alzheimer's disease. Neurology. 1998:51:351-7.

13. Wang DS, Bennett DA, Mufson E, Cochran E, Dickson DW. Decreases in soluble alpha-synuclein in frontal cortex correlate with cognitive decline in the elderly. Neurosci Lett. 2004;359:104-8. 
14. Parkkinen $L$, Soininen $H$, Alafuzoff I. Regional distribution of alpha-synuclein pathology in unimpaired aging and Alzheimer disease. J Neuropathol Exp Neurol. 2003;62:363-7.

15. Sonnen J, Larson EB, Crane PK, Haneuse S, Li G, Schellenberg GD, et al. Pathological correlates of dementia in a longitudinal, population-based sample of aging. Ann Neurol. 2007;62:406-13.

16. Jellinger KA. Age-associated prevalence and risk factors of Lewy body pathology in a general population. Acta Neuropathol. 2003;106:383-4.

17. Xuereb JH, Brayne C, Dufouil C, Gertz H, Wischik C, Harrington C, et al. Neuropathological findings in the very old. Results from the first 101 brains of a population-based longitudinal study of dementing disorders. Ann NY Acad Sci. 2000;903:490-6.

18. White L, Petrovitch H, Hardman J, Nelson J, Davis DG. Cerebrovascular pathology and dementia in autopsied Honolulu-Asia Aging Study participants. Ann NY Acad Sci. 2002;977:9-23.

19. McKeith I, Mintzer J, Aarsland D, Burn D, Chiu H, Cohen-Mansfield J, et al Dementia with Lewy bodies. Lancet Neurol. 2004;3:19-28.

20. Zaccai J, Ince P, Brayne C. Population-based neuropathological studies of dementia: design, methods and areas of investigation - a systematic review. BMC Neurol. 2006;6:2.

21. Cognitive function and dementia in six areas of England and Wales: the distribution of MMSE and prevalence of GMS organicity level in the MRC CFA Study. The Medical Research Council Cognitive Function and Ageing Study (MRC CFAS). Psychol Med. 1998;28:319-35

22. Folstein MF, Folstein SE, McHugh PR. "Mini-mental state". A practical method for grading the cognitive state of patients for the clinician. J Psychiatr Res. 1975;12:189-98.

23. Copeland JR, Dewey ME, Griffiths-Jones HM. A computerized psychiatric diagnostic system and case nomenclature for elderly subjects: GMS and AGECAT. Psychol Med. 1986;16:89-99.

24. Roth M, Tym E, Mountjoy CQ, Hendrie H, Verma S, Goddard R. CAMDEX. A standardised instrument for the diagnosis of mental disorder in the elderly with special reference to the early detection of dementia. Br J Psychiatry. 1986;149:698-709.

25. Braak H, Braak E, Yilmazer D, de Vos RA, Jansen EN, Bohl J, et al. Amygdala pathology in Parkinson's disease. Acta Neuropathol. 1994;88:493-500.

26. Braak H, Braak E. Pathoanatomy of Parkinson's disease. J Neurol. 2000;247:|13-||10.

27. Del Tredici K, Rub U, De Vos RA, Bohl JR, Braak H. Where does parkinson disease pathology begin in the brain? J Neuropathol Exp Neurol. 2002;61:413-26.

28. Parkkinen L, Kauppinen T, Pirttila T, Autere JM, Alafuzoff I. Alpha-synuclein pathology does not predict extrapyramidal symptoms or dementia. Ann Neurol. 2005:57:82-91.

29. Saito Y, Kawashima A, Ruberu NN, Nyoka N, Fujiwara H, Koyama S, et al. Accumulation of phosphorylated alpha-synuclein in aging human brain. J Neuropathol Exp Neurol. 2003;62:644-54.

30. McKeith IG, Galasko D, Kosaka K, Perry EK, Dickson DW, Hansen LA, et al. Consensus guidelines for the clinical and pathologic diagnosis of dementia with Lewy bodies (DLB): report of the consortium on DLB international workshop. Neurology. 1996:47:1113-24.

31. Zaccai J, Brayne C, McKeith I, Matthews F, Ince PG. Patterns and stages of alpha-synucleinopathy: Relevance in a population-based cohort. Neurology. 2008;70:1042-8.

32. Kosaka K, Iseki E, Odawara T, Yamamoto T. Cerebral type of Lewy body disease. Neuropathology. 1996;16:2-35.

33. Hamilton RL. Lewy bodies in Alzheimer's disease: a neuropathological review of 145 cases using alpha-synuclein immunohistochemistry. Brain Pathol. 2000;10:378-84

34. Uchikado H, Lin WL, DeLucia MW, Dickson DW. Alzheimer disease with amygdala Lewy bodies: a distinct form of alpha-synucleinopathy. J Neuropathol Exp Neurol. 2006;65:685-97.

35. Marui W, Iseki E, Nakai T, Miura S, Kato M, Ueda K, et al. Progression and staging of Lewy pathology in brains from patients with dementia with Lewy bodies. J Neurol Sci. 2002;195:153-9.

36. Popescu A, Lippa CF, Lee VM, Trojanowski JQ. Lewy bodies in the amygdala: increase of alpha-synuclein aggregates in neurodegenerative diseases with tau-based inclusions. Arch Neurol. 2004;61:1915-9.

37. Kosaka K. Diffuse Lewy body disease. Rinsho Shinkeigaku. 1995;35:1455-6.

38. Jellinger KA. A critical evaluation of current staging of alpha-synuclein pathology in Lewy body disorders. Biochim Biophys Acta. 2009;1792:730-40.
39. Foltynie T, Matthews FE, Ishihara L, Brayne C, MRC CFAS. The frequency and validity of self-reported diagnosis of Parkinson's Disease in the UK elderly: MRC CFAS cohort. BMC Neurol. 2006;6:29.

40. Beach TG, White 3rd CL, Hladik CL, Sabbagh MN, Connor DJ, Shill HA, et al. Olfactory bulb alpha-synucleinopathy has high specificity and sensitivity for Lewy body disorders. Acta Neuropathol. 2009;117:169-74.

\section{Submit your next manuscript to BioMed Central and take full advantage of:}

- Convenient online submission

- Thorough peer review

- No space constraints or color figure charges

- Immediate publication on acceptance

- Inclusion in PubMed, CAS, Scopus and Google Scholar

- Research which is freely available for redistribution 